\title{
Association of CTXN3-SLC12A2 polymorphisms and schizophrenia in a Thai population
}

\author{
Benjaporn Panichareon ${ }^{1 \dagger}$, Kazuhiro Nakayama ${ }^{2 \dagger}$, Sadahiko Iwamoto², Wanpen Thurakitwannakarn ${ }^{3}$ and \\ Wasana Sukhumsirichart ${ }^{*}$
}

\begin{abstract}
Background: A genome-wide association study (GWAS) combined with brain imaging as a quantitative trait analysis revealed that the SNPs near CTXN3-SLC12A2 region were related to forebrain development and stress response which involved in schizophrenia. In the present study, the SNPs in this region were analyzed for association with schizophrenia in a Thai population.
\end{abstract}

Methods: A total of 115 schizophrenia and 173 unrelated normal controls with mean age of $37.87 \pm 11.8$ and $42.81 \pm 6.0$ years, respectively, were included in this study. Genotyping was performed using polymerase chain reaction and high-resolution melting (HRM) analysis. The difference in genotype distribution between patient and control was assessed by Chi-square test of the SPSS software.

Results: We found a significant association between the GWAS-discovered SNP, rs245178, with the risk of schizophrenia in the Thai population $[P=0.006$, odds ratio for the minor $\mathrm{G}$ allele: $0.62(0.46-0.83)]$. Additionally, another potential SNP, rs698172, which was in moderate linkage disequilibrium with rs245178, also showed strong association with schizophrenia $[P=0.003$, odds ratio for minor T allele: $0.61(0.46-0.82)]$. This association remained significant at 5\% level after the Bonferroni correction for multiple testing.

Conclusions: This study shows that two SNPs in intergenic of the CTXN3 and SLC12A2 genes, rs245178 and rs698172, are associated with risk of schizophrenia in Thai population. Further study is required for clarification the role of genetic variation around these SNPs in expression pattern of the CTXN3 and SLC12A2 genes, which may be involved in schizophrenia pathogenesis.

Keywords: Schizophrenia, Single nucleotide polymorphisms, CTXN3, SLC12A2, High- resolution melting analysis

\section{Background}

Schizophrenia is characterized by abnormalities in perception, thought, cognition, and expression of reality. It is clinically described by positive and negative symptoms [1]. Positive symptoms are psychotic behaviors that are present in schizophrenia but absent in healthy individuals, include hallucinations, delusions, and thought disorder. Negative symptoms are factors that absent in schizophrenia patients but are present in healthy persons [2]. Schizophrenia is one of the most common psychiatric disorders and its prevalence is $1 \%$ of the worldwide

\footnotetext{
* Correspondence: wasanas@swu.ac.th

${ }^{\dagger}$ Equal contributors

'Department of Biochemistry, Faculty of Medicine, Srinakharinwirot University, Bangkok 10110, Thailand

Full list of author information is available at the end of the article
}

population [3]. Twin and family studies suggest that genetic factors play a major role in schizophrenia $[4,5]$.

A variety of genetic studies reported several chromosomal regions associated with schizophrenia and related phenotypes. A quantitative trait locus for schizophrenia was mapped to a chromosome 5 region in Americans of European ancestry [6]. A meta-analysis of European ethnicity showed that the 5q23.2-q34 region was significantly linked with schizophrenia [7].

A genome wide association study (GWAS) in combination with the data from brain imaging of dorsolateral prefrontal cortex (DLPFC) as quantitative trait (QT) analysis revealed 6 genes/regions that involved in neurodevelopment and response to stress. The first three genes including ROBO1-ROBO2, TNIK, and CTXN3-SLC12A2 were identified by having 2 single-nucleotide polymorphisms (SNPs) each significant at $P<10^{-6}$ for the interaction

\section{() Biomed Central}


between the imaging QT and the diagnosis. The other three genes including POU3F2, TRAF, and GPC1 had a significant SNP at $<10^{-6}$ [8]. Because the GWAS was a screening method over the whole genome to identify the SNPs related to risk for schizophrenia and the subject of previous study mostly Caucasian ethnic group [8]. Moreover, there is no association study of those genes and risk of schizophrenia in other ethnic groups rather than European ancestry. A replication study with Asian ethnic groups would help to reinforce the signals obtained in a European GWAS. Furthermore, the testing of a population showing different linkage disequilibrium (LD) structures may assist in identifying the true causative variant in the genomic region of interest.

In Thailand, the prevalence of schizophrenia is comparable with the worldwide value (approximately 1\%). Genetic variations associated with risks of schizophrenia have been investigated in opioid-binding protein/cell adhesion molecule (OPCML) gene [9] using GWAS-discovered SNPs of Europeans ancestry [10]. There was strong association between an intronic SNP of the $O P C M L$ gene (rs1784519) and the risk of schizophrenia in a Thai population $[P=0.00036$, odds ratio for the minor A allele: 2.11(1.57-2.84)] [9].

This study aims to investigate the association between SNPs in CTXN3-SLC12A2 region with the risk of schizophrenia in the Thai population. The CTXN3 gene is located on 5q23 encode CTXN3 (cortexin 3), a brain specific integral membrane protein highly enriched in cortex. It is expressed in fetal brain and increases in density perinatally. The gene consists of 3 exons and spans an approximate $9.6 \mathrm{~kb}$ region. It has 2 alternative transcript variants expressed in the brain and kidneys, and encodes an 81amino acids protein [11]. This gene is highly conserved in various species, suggesting that this gene plays important roles in brain and kidney function. The gene, SLC12A2 (solute carrier family 12 , member 2 ), found in the same region as CTXN3, encodes a sodium-potassium-chloride $(\mathrm{Na}-\mathrm{K}-\mathrm{Cl})$ cotransporter protein which is expressed in various human tissues, including the brain [12]. SLC12A2 is involved in the regulation of GABA neurotransmission. Both CTXN3 and SLC12A2 are indicated to play a role in normal brain function, in addition, the CTXN3-SLC12A2 region is considered as the second most important region linked to schizophrenia in the meta-analysis $[7,8]$, therefore, they may be involved in schizophrenia pathogenesis. This study, the SNPs in the intergenic of CTXN3-SLC12A2 region, 3 '-UTR and intronic of CTXN3 gene were analyze for their association with schizophrenia.

\section{Methods}

Subjects

A total number of subjects consisted of 115 schizophrenia patients (61 males and 54 females) with a mean age of $37.87 \pm 11.8$ years and 173 unrelated healthy controls (110 males and 63 females) with a mean age of $42.81 \pm 6.0$ years. The patients were diagnosed according to the Diagnostic and Statistical Manual of Mental Disorders, Fourth Edition (DSM-IV) criteria. The healthy controls were also psychiatrically screened to confirm that they did not suffer from a psychiatric disorder. All patients and healthy controls gave informed consent for participation in this study, which was approved by the ethical committee at the Faculty of Medicine, Chiang Mai University and Faculty of Medicine, Srinakharinwirot University.

\section{Genotyping}

Genomic DNAs of patient and control were extracted from whole blood $(5 \mathrm{ml}$ ) using Flexigene DNA kit (Qiagen, German). Genotyping was performed by using polymerase chain reaction (PCR) and followed by highresolution melting analysis (HRM). PCR primers were designed using LightScanner Primer Design software (Idaho Technology, Salt Lake City, UT, USA) and the primer sequences were included in supplement table (available online). The PCR was carried out using TaKaRa $E x$ Taq Hot Start Version (TaKaRa, Tokyo, Japan) with LCGreen + (Idaho Technology). The high-resolution melting analysis was performed using a LightScanner (Idaho Technology, USA). Sequences of primers are provided in the Additional file 1.

\section{Statistical analysis}

The linkage disequilibrium (LD) status of the GWASdiscovered marker SNPs; rs245178, rs245201 [8], and nearby SNPs; rs1421746, rs711360, rs698171, rs698172, rs245314, rs245311, rs245310, rs9285907, rs151849, rs6874357, rs245199, rs245195, rs245192, rs245191, rs1579284, rs181746, rs245186, rs6864687, rs151879, rs245179, rs245181, and rs245183, were determined using 47 randomly selected schizophrenia patients and healthy controls. LD analyses were performed using Haploview software [13]. SNPs with a marked deviation from Hardy-Weinberg equilibrium $(P<0.01)$ were excluded from further analysis. The association analysis was assessed by using Pearson's Chi-square test implemented in SPSS program version 11.5 for Window. We assessed 3 genotype models (additive, dominant, and recessive) in the association analysis. The significance level of the association test was set at 5\% and moreover, we also applied the Bonferroni correction for the numbers of SNPs tested. For the SNPs showing significant association, mode of inheritance was inferred by using the method proposed Zintzaras and Santos [14]. The generalized odds ratio of genotypes [15] and odds ratio of the minor allele of each SNP were calculated. For the allelic test, we evaluated statistical power by using the G*Power 
3 [16]. Odds ratios that ensured $80 \%$ power $(P<0.05)$ were estimated as $2.00(0.37), 1.74(0.51), 1.65(0.57), 1.62$ $(0.60)$, and $1.62(0.61)$, for alleles with frequencies in the controls of $0.1,0.2,0.3,0.4$ and 0.5 , respectively. We also estimated sex and age adjusted-odds ratio of the SNP genotypes with the aid of multiple logistic regression analysis. Haplotypes of the SNPs were reconstructed by using PHASE2.0 [17]. Differences in haplotype frequencies between the patients and the controls were assessed by using $X^{2}$ test.

\section{Results}

\section{Association study of SNPs and schizophrenia}

We first tested the associations between the GWAS-discovered SNPs that located in the intergenic of CTXN3SLC12A2 region, 3'-UTR and intronic of CTXN3 gene with schizophrenia in Thai population. The result of the association analysis is summarized in Table 1. The SNPs in intergenic, rs245178, showed significant differences in the genotype distribution between patients and controls $(P=0.006$, under the dominant model). The odds ratio
(OR) and 95\% confidence intervals (CI) of the minor G allele were estimated to be 0.62 and $0.46-0.83$, respectively. The lower prevalence of the $\mathrm{G}$ allele in patients was consistent with the result of the previous report [8]. In contrast, another GWAS-discovered SNP, rs245201, did not show significant differences in genotype distribution in Thai population $(P>0.05)$.

\section{Linkage disequilibrium analysis}

The LD status of rs 245178 and nearby SNPs in 47 randomly selected Thai individuals was determined and the result of LD analysis is shown in Figure 1. The LD structure was generally similar to that of a population with European ancestry (HapMap CEU, data not shown). The rs245178 was found in a large LD block spanning $43 \mathrm{~kb}$ in our Thai population. To narrow the region containing the responsible SNPs, we selected the rs1421746, rs698171, and rs698172, which were in moderate LD with the rs245178, and tested for the association with schizophrenia. The rs698172 showed the strongest association $(P=0.003$, under the dominant model) and the

Table 1 Results of the association analyses

\begin{tabular}{|c|c|c|c|c|c|c|c|c|c|}
\hline \multirow{2}{*}{$\frac{\text { dbSNP ID }}{\text { rs77111139 }}$} & \multirow{2}{*}{$\begin{array}{l}\text { Type of SNP } \\
\text { CTXN3 }\end{array}$} & & \multicolumn{3}{|c|}{ Genotype frequencies } & \multirow[t]{2}{*}{ MAF $^{1}$} & \multicolumn{2}{|l|}{$P^{2}$} & \multirow{2}{*}{$\begin{array}{l}\text { Allelic } \text { OR }^{3} \text { Generalized OF } \\
1.29(0.96-1.74)\end{array}$} \\
\hline & & & A/A & $A / G$ & $\mathrm{G} / \mathrm{G}$ & & a & 0.139 & \\
\hline & intronic & Patients & 17 & 45 & 52 & 0.34 & $d$ & 0.528 & $1.23(0.84-1.95)$ \\
\hline & & Controls & 13 & 74 & 85 & 0.29 & $r$ & 0.047 & \\
\hline \multirow[t]{3}{*}{ rs248707 } & CTXN3 & & $\mathrm{C} / \mathrm{C}$ & $\mathrm{C} / \mathrm{T}$ & $\mathrm{T} / \mathrm{T}$ & & a & 0.594 & $1.24(0.88-1.74)$ \\
\hline & 3'UTR & Patients & 7 & 40 & 67 & 0.24 & $d$ & 0.325 & $1.27(0.80-2.00)$ \\
\hline & & Controls & 8 & 53 & 111 & 0.20 & $r$ & 0.580 & \\
\hline \multirow[t]{3}{*}{ rs1421746 } & Intergenic & & $\mathrm{C} / \mathrm{C}$ & $\mathrm{C} / \mathrm{T}$ & $\mathrm{T} / \mathrm{T}$ & & a & 0.025 & $0.61(0.46-0.81)$ \\
\hline & & Patients & 37 & 49 & 26 & 0.45 & $d$ & 0.038 & $0.58(0.39-0.86)$ \\
\hline & & Controls & 38 & 71 & 64 & 0.57 & $r$ & 0.015 & \\
\hline \multirow[t]{3}{*}{ rs698172 } & Intergenic & & $\mathrm{C} / \mathrm{C}$ & $\mathrm{C} / \mathrm{T}$ & $\mathrm{T} / \mathrm{T}$ & & a & 0.012 & $0.61(0.46-0.82)$ \\
\hline & & Patients & 49 & 41 & 21 & 0.38 & $d$ & $0.003^{*}$ & $0.58(0.38-0.87)$ \\
\hline & & Controls & 47 & 81 & 45 & 0.49 & $r$ & 0.167 & \\
\hline \multirow[t]{3}{*}{ rs698171 } & Intergenic & & $\mathrm{G} / \mathrm{G}$ & $\mathrm{G} / \mathrm{T}$ & $\mathrm{T} / \mathrm{T}$ & & a & 0.028 & $1.56(1.13-2.16)$ \\
\hline & & Patients & 57 & 44 & 11 & 0.29 & $d$ & 0.10 & $1.58(1.01-2.48)$ \\
\hline & & Controls & 105 & 63 & 5 & 0.21 & $r$ & 0.01 & \\
\hline \multirow[t]{3}{*}{ rs245201 } & Intergenic & & A/A & $A / G$ & $\mathrm{G} / \mathrm{G}$ & & a & 0.083 & $0.81(0.60-1.08)$ \\
\hline & & Patients & 51 & 41 & 22 & 0.37 & $d$ & 0.052 & $0.77(0.51-1.15)$ \\
\hline & & Controls & 56 & 82 & 30 & 0.42 & $r$ & 0.759 & \\
\hline \multirow[t]{3}{*}{ rs 245178} & Intergenic & & A/A & $\mathrm{A} / \mathrm{G}$ & $\mathrm{G} / \mathrm{G}$ & & a & 0.021 & $0.62(0.46-0.83)$ \\
\hline & & Patients & 54 & 43 & 15 & 0.33 & d & $0.006^{*}$ & $0.57(0.38-0.86)$ \\
\hline & & Controls & 55 & 83 & 34 & 0.44 & r & 0.165 & \\
\hline
\end{tabular}

${ }^{1}$ MAF: Minor allele frequency.

${ }^{2} P$ values for the additive model (a), dominant model (d), and recessive model $(r)$ are shown. Asterisks indicate tests that were significant at $5 \%$ level after the Bonferroni correction for multiple SNP testing.

${ }^{3}$ Allelic OR (upper) indicates odds ratio and $95 \%$ confidence interval of minor alleles. Generalized OR (lower) indicates odds ratio and $95 \%$ confidence interval of genotypes [14]. 


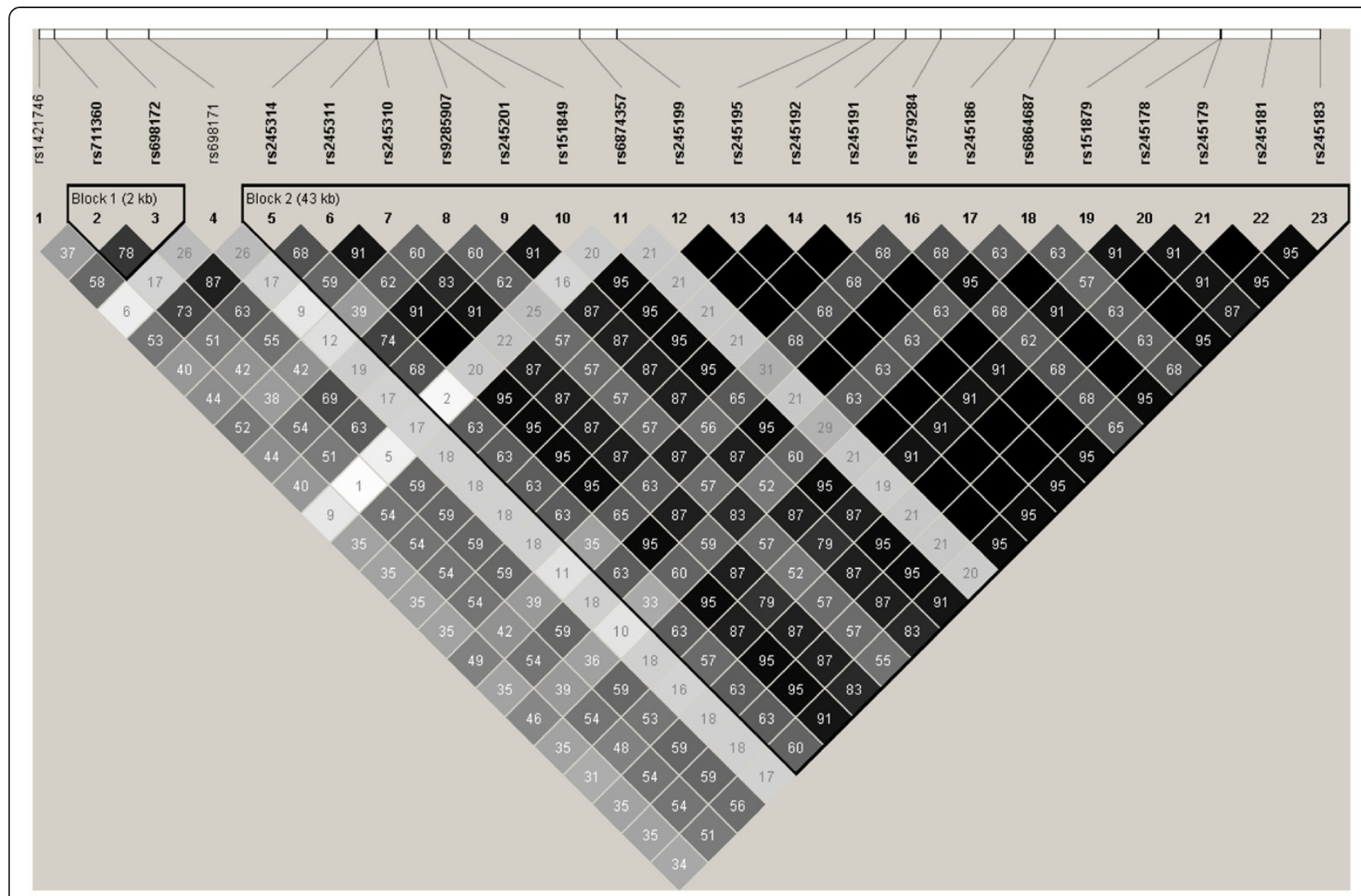

Figure 1 Linkage disequilibrium status of the CTXN3-SLC12A2 region in Thai individuals. $r^{2}$ values calculated from genotype data of 47 Thai are indicated. The haplotype blocks were defined by using the default setting of the Haploview software.

minor $\mathrm{T}$ allele was found to have lower frequency in patients than in controls $(\mathrm{OR}=0.61)$ (Table 1$)$. Sex and age adjusted- OR and $95 \% \mathrm{CI}$ of carriers of the each minor allele were estimated as 0.50 and $0.30-0.84$ for both SNPs. We also tested the rs248707, rs6959787, and rs7711139, which were located in the CTXN3 gene and did not show strong LD with the SNPs tested above. The rs248707 and rs7711139, which possibly had functional consequences for the CTXN3 gene, did not show a significant association with schizophrenia $(P=0.325$ and $P=0.528$, respectively). Marked deviation from Hardy-Weinberg equilibrium was observed for the rs6959787 $(P=0.004$ in the patients; $P=0.0009$ in the controls) and thus, association analysis was not performed for this SNP. Among the seven SNPs tested, the rs 245178 and rs698172 remained significant at 5\% level after the Bonferroni correction for multiple SNP testing $(P<0.0071)$. Degree of dominance $h$ for rs245178 and rs698172 were -0.50 and -0.51 , respectively. These values fell into $0<|h|<1$, supporting that the minor allele of these two SNPs were dominant [14]. LD status of the 7 SNPs in the patients and in the controls are shown in Figure 2. Neither $D^{\prime}$ nor $r^{2}$ values showed striking differences between the patients and controls.

\section{Haplotype analysis}

We reconstructed haplotypes of rs 245178 and rs698172 and tested the differences in haplotype frequencies between patients and controls (Table 2). A total of four haplotypes were observed in the studied population. Frequencies of these haplotypes were significantly different between the patients and the controls $(P=0.0447)$. Moreover, a haplotype consist $\mathrm{T}$ allele of rs698172 and $\mathrm{G}$ allele of rs245178 was significantly underrepresented in patients (in Table 2; Haplotype 2 versus Haplotype 3, $P=0.005$, OR $=0.58$ ).

\section{Discussion}

In the present study, a total of four SNPs in CTXN3SLC12A2 region-rs245178, rs698172, rs1421746, and rs698171 were shown to be significantly associated with a risk of schizophrenia in a Thai population that is consistent with the previous GWAS report [8]. Another GWAS-discovered SNP, rs245201, showed a marginal association with schizophrenia $(P=0.052$, under the dominant model). In a population of European ancestry (HapMap CEU panel), rs245201 was in absolute LD with rs698172, but this was not the case in our Thai population $\left(r^{2}=0.5\right)$. The differences in LD structure would 


\begin{tabular}{|l|c|c|c|c|c|c|c|}
\hline Patiens & rs7711139 & rs248707 & rs1421746 & rs698172 & rs698171 & rs245201 & rs245178 \\
\hline rs7711139 & & 0.507 & 0.661 & 0.722 & 0.447 & 0.472 & 0.723 \\
\hline rs248707 & 0.042 & & 0.504 & 0.351 & 0.552 & 0.499 & 0.703 \\
\hline rs1421746 & 0.181 & 0.063 & & 0.953 & 0.461 & 0.633 & 0.771 \\
\hline rs698172 & 0.159 & 0.023 & 0.674 & & 0.941 & 0.627 & 0.788 \\
\hline rs698171 & 0.043 & 0.221 & 0.072 & 0.220 & & 0.654 & 0.926 \\
\hline rs245201 & 0.069 & 0.047 & 0.299 & 0.394 & 0.110 & & 0.884 \\
\hline rs245178 & 0.132 & 0.076 & 0.362 & 0.509 & 0.171 & 0.642 & \\
\hline
\end{tabular}

\begin{tabular}{|c|c|c|c|c|c|c|c|}
\hline Controls & rs 7711139 & rs248707 & rs 1421746 & rs698172 & rs698171 & rs 245201 & rs245178 \\
\hline rs7711139 & & 0.738 & 0.771 & 0.785 & 0.469 & 0.856 & 0.767 \\
\hline rs248707 & 0.056 & $=$ & 0.290 & 0.171 & 0.221 & 0.409 & 0.444 \\
\hline rs 1421746 & 0.330 & 0.029 & Tis & 0.985 & 0.380 & 0.844 & 0.799 \\
\hline rs698172 & 0.244 & 0.007 & 0.701 & r & 0.959 & 0.867 & 0.799 \\
\hline rs698171 & 0.024 & 0.045 & 0.052 & 0.240 & & 0.946 & 0.901 \\
\hline rs245201 & 0.224 & 0.030 & 0.401 & 0.598 & 0.182 & & 0.935 \\
\hline rs245178 & 0.190 & 0.039 & 0.372 & 0.517 & 0.171 & 0.822 & \\
\hline
\end{tabular}

\begin{tabular}{|c|c|c|c|c|c|}
\hline$D^{\prime}$ & LOD $<2$ & $L O D \geq 2$ & & & \\
\hline$r^{\wedge} 2$ & $<0.2$ & $\geq 0.2$ & $\geq 0.4$ & $\geq 0.6$ & $\geq 0.8$ \\
\hline
\end{tabular}

Figure 2 Pairwise LD status of the 7 SNPs in 115 patients and 173 controls. Upper and lower triangles indicate $D^{\prime}$ and $r^{2}$, respectively. LD status of each pair of SNPs is color-coded by its strength (see the explanatory notes in the figure).

explain the apparent contradiction between these studies. Furthermore, the rs698172, which was separated by $45.5 \mathrm{~kb}$ from the rs 245178 , showed the strongest association with risk of schizophrenia in the Thai group. The minor $\mathrm{T}$ allele of rs698172 was shown to reduce the risk of schizophrenia. The haplotype-based association analysis revealed that the frequency of haplotypes of the rs698172 $\mathrm{T}$ and $\mathrm{rs} 245178 \mathrm{G}$ alleles was significantly lower in patients $(P=0.005)$, and the odds ratio was smaller than those of rs698172 and rs245178. Further verification is required to determine whether this haplotype effect is the actual influences susceptibility to schizophrenia. The associations detected for the rs 245178 and rs698172 were considered to simply reflect LD of these two SNPs. The rs245178 and rs698172 were located in the intergenic region between CTXN3 and SLC12A2 genes, and it is still

Table 2 Haplotype analysis

\begin{tabular}{lllll}
\hline & rs698172 & rs245178 & Patient & Control \\
\hline Haplotype1 & $\mathrm{T}$ & $\mathrm{A}$ & $0.09(0.02)$ & $0.10(0.02)$ \\
Haplotype2 & $\mathrm{T}$ & $\mathrm{G}$ & $0.29(0.03)$ & $0.39(0.03)$ \\
Haplotyep3 & $\mathrm{C}$ & $\mathrm{A}$ & $0.58(0.03)$ & $0.46(0.03)$ \\
Haplotype4 & $\mathrm{C}$ & $\mathrm{G}$ & $0.04(0.01)$ & $0.04(0.01)$ \\
& $\boldsymbol{P}^{*}$ & $\mathbf{O R} \mathbf{( 9 5 \% \mathbf { C l } )}$ & & \\
Overall & 0.04 & & & \\
Haplotype 2 versus & 0.005 & $0.58(0.43-0.79)$ & & \\
Haplotype 3 & & & &
\end{tabular}

* $P$ values in $x^{2}$ test are shown.

For each haplotype, relative frequencies and the standard deviation (in parentheses) are shown. unclear whether functional elements affecting gene functions exist in this region.

CTXN3 protein is a brain-specific integral membrane protein, highly enriched within the cortex [18]. It shows $43 \%$ homology with cortexin (CTXN 1), which is highly enriched in the cerebral cortex and is probably an integral membrane protein playing a role in the developing and adult cerebral cortex [18]. It is expected that CTXN3 is also a member of the integral membrane proteins and is relevant to the development and function of the cerebral cortex. Another candidate gene in this region, SLC12A2, was expressed in various human tissues, including brain [12]. SLC12A2 was highly expressed in developing cortical neurons and promotes the accumulation of chloride ion $\left(\mathrm{Cl}^{-}\right)$in neurons [19]. Moreover, SLC12A2 protein facilitates seizure in the developing brain [19]. The significant associations between SNPs and risk of schizophrenia suggest potential roles of these two genes in the pathogenesis of schizophrenia.

\section{Conclusions}

The present study showed that two SNPs in the CTXN3-SLC12A2 region, rs245178, and rs698172 are associated with risk of schizophrenia in Thai population. These SNPs were located in intergenic region with unknown function. Further study, it would be of interest to identify genetic variation around these SNPs affecting the expression pattern of the CTXN3 and SLC12A2 genes and test the association with risk for schizophrenia. 


\section{Additional file}

Additional file 1: Primers for high-resolution melting analysis

\section{Competing interests}

The authors declare that they have no competing interests.

\section{Acknowledgements}

This study was supported by the Royal Golden Jubilee Ph.D. Program of the Thailand Research Fund (PHD/0213/2551); Srinakharinwirot University (149/ 2550, 218/2552); KAKENHI 21687021. This study was also subsidized by JKA through its promotion funds from KEIRIN RACE. The study sponsors played no role in the study design; in the collection, analysis and interpretation of data; in the writing of the report; or the decision to submit for the paper.

\section{Author details}

${ }^{1}$ Department of Biochemistry, Faculty of Medicine, Srinakharinwirot University, Bangkok 10110, Thailand. 'Division of Human Genetics, Center for Molecular Medicine, Jichi Medical University, Tochigi 329-0498, Japan. ${ }^{3}$ Department of Psychiatry, Faculty of Medicine, Srinakharinwirot University, Bangkok 10110, Thailand.

\section{Authors' contributions}

BP and KN participated in the study design, performed the genetic studies, statistical analysis and prepared draft of the manuscript. SI gave critical comments on the manuscript. WT investigated and diagnosed the control and patient according to DSM-IV criteria. WS participated in study design, concluded the results, and revised manuscript. All authors read and approved the final manuscript.

Received: 26 September 2011 Accepted: 29 May 2012

Published: 29 May 2012

\section{References}

1. Andreasen NC, Flaum M: Schizophrenia: the characteristic symptoms. Schizophr Bull 1991, 17:27-49.

2. Tandon R, Nasrallah HA, Keshavan MS: Schizophrenia, "just the facts" 4 . Clinical features and conceptualization. Schizophr Res 2009, 110:1-23.

3. Cardno AG, Marshall EJ, Coid B, Macdonald AM, Ribchester TR, Davies NJ, Venturi P, Jones LA, Lewis SW, Sham PC, et al: Heritability estimates for psychotic disorders: the Maudsley twin psychosis series. Arch Gen Psychiatry 1999, 56:162-168.

4. Dworkin RH, Lenzenweger MF: Symptoms and the genetics of schizophrenia: implications for diagnosis. Am J Psychiatry 1984, 141:15411546.

5. Kendler KS, Diehl SR: The genetics of schizophrenia: a current, geneticepidemiologic perspective. Schizophr Bull 1993, 19:261-285.

6. Almasy L, Gur RC, Haack K, Cole SA, Calkins ME, Peralta JM, Hare E, Prasad K, Pogue-Geile MF, Nimgaonkar V, Gur RE: A genome screen for quantitative trait loci influencing schizophrenia and neurocognitive phenotypes. Am J Psychiatry 2008, 165:1185-1192.

7. Lewis CM, Levinson DF, Wise LH, DeLisi LE, Straub RE, Hovatta I, Williams NM, Schwab SG, Pulver AE, Faraone SV, et al: Genome scan meta-analysis of schizophrenia and bipolar disorder, part II: Schizophrenia. Am J Hum Genet 2003, 73:34-48.

8. Potkin SG, Turner JA, Guffanti G, Lakatos A, Fallon JH, Nguyen DD, Mathalon D, Ford J, Lauriello J, Macciardi F: A genome-wide association study of schizophrenia using brain activation as a quantitative phenotype. Schizophr Bull 2009, 35:96-108.

9. Panichareon B, Nakayama K, Thurakitwannakarn W, Iwamoto S, Sukhumsirichart W: OPCML Gene as a Schizophrenia Susceptibility Locus in Thai Population. J Mol Neurosci 2012, 46:373-377.

10. O'Donovan MC, Craddock N, Norton N, Williams H, Peirce T, Moskvina V, et al: Identification of loci associated with schizophrenia by genome-wide association and follow-up. Nat Genet 2008, 40:1053-1055.

11. Wang HT, Chang JW, Guo Z, Li BG: In silico-initiated cloning and molecular characterization of cortexin 3, a novel human gene specifically expressed in the kidney and brain, and well conserved in vertebrates. Int J Mol Med 2007, 20:501-510.
12. Payne JA, Xu JC, Haas M, Lytle CY, Ward D, Forbush B 3rd: Primary structure, functional expression, and chromosomal localization of the bumetanide-sensitive Na-K-Cl cotransporter in human colon. J Biol Chem 1995, 270:17977-17985.

13. Barrett JC, Fry B, Maller J, Daly MJ: Haploview: analysis and visualization of LD and haplotype maps. Bioinformatics 2005, 21:263-265.

14. Zintzaras E, Santos M: Estimating the mode of inheritance in genetic association studies of qualitative traits based on the degree of dominance index. BMC Med Res Methodol 2011, 11:171-182.

15. Zintzaras E: The Generalized Odds Ratio as a Measure of Genetic Risk Effect in the Analysis and Meta-Analysis of Association Study. Stat App Genet Mol Biol 2010, 9:Article 21.

16. Faul F, Erdfelder $E$, Lang AG, Buchner A: $G^{*} 3$ Power 3: a flexible statistical power analysis program for the social, behavioral, and biomedical sciences. Behav Res Methods 2007, 39:175-191.

17. Stephens M, Donnelly P: A comparison of bayesian methods for haplotype reconstruction from population genotype data. Am J Hum Genet 2003, 73:1162-1169.

18. Coulter PM 2nd, Bautista EA, Margulies JE, Watson JB: Identification of cortexin: a novel, neuron-specific, 82-residue membrane protein enriched in rodent cerebral cortex. J Neurochem 1993, 61:756-759.

19. Dzhala VI, Talos DM, Sdrulla DA, Brumback AC, Mathews GC, Benke TA, Delpire $E$, Jensen FE, Staley KJ: NKCC1 transporter facilitates seizures in the developing brain. Nat Med 2005, 11:1205-1213.

doi:10.1186/1744-9081-8-27

Cite this article as: Panichareon et al:: Association of CTXN3-SLC12A2 polymorphisms and schizophrenia in a Thai population. Behavioral and Brain Functions 2012 8:27.

\section{Submit your next manuscript to BioMed Central and take full advantage of:}

- Convenient online submission

- Thorough peer review

- No space constraints or color figure charges

- Immediate publication on acceptance

- Inclusion in PubMed, CAS, Scopus and Google Scholar

- Research which is freely available for redistribution

Submit your manuscript at www.biomedcentral.com/submit
C) Biomed Central 